\title{
Liderazgo del docente universitario y el rendimiento académico
}

\author{
Mario Villegas Yarleque \\ ORCID: https://orcid.org/0000-0001-5572-1372 \\ mvillegas@unf.edu.pe \\ Universidad Nacional de Frontera \\ Sullana-Perú
}

Cynthia Milagros Apaza Panca

ORCID: https://orcid.org/0000-0002-5524-2627

capaza@unf.edu.pe

Universidad Nacional de Frontera

Sullana-Perú

\author{
Gretel Fiorella Villegas Aguilar \\ ORCID: https://orcid.org/0000-0001-8145-1003 \\ gretel.villegas@upn.pe \\ Universidad Privada del Norte \\ Lima-Perú
}

\author{
Susana Soledad Chinchay Villarreyes \\ ORCID: https://orcid.org/0000-0001-8348-4490 \\ schinchay@unf.edu.pe \\ Universidad Nacional de Frontera \\ Sullana-Perú
}

Priscila E. Luján-Vera

ORCID: https://orcid.org/0000-0002-1359-5715

priscilavera930@gmail.com

Universidad Nacional de Frontera

Sullana-Perú

Recibido (04/02/21), Aceptado (23/02/21)

\begin{abstract}
Resumen:El propósito de la investigación fue determinar la relación entre el estilo de liderazgo de los docentes y el rendimiento académico de los estudiantes del IV ciclo de la Facultad de Administración Hotelera y de Turismo de la Universidad Nacional de Frontera-Perú. La investigación fue de tipo analítico, ambispectivo y de diseño no experimental, transversal, correlacional. La Recolección de datos se realizó a través del uso de técnicas de observación y la encuesta, con sus instrumentos: fichas técnicas de observación y cuestionario; respectivamente. La muestra de estudio estuvo representada por 14 estudiantes. Para el análisis estadístico se empleó las medidas de tendencia central como es la media aritmética, la varianza y la desviación estándar. Además, se empleó la estadística inferencial, el coeficiente Rho de Spearman, para medir la correlación entre la variable de liderazgo del docente y el rendimiento académico de los estudiantes. Los resultados arrojaron que los docentes aplican el estilo de liderazgo democrático obteniendo en los estudiantes un rendimiento académico promedio de 13.26 como aceptable y el coeficiente Rho $=-0.44$ que indica la existencia de una correlación media negativa entre las variables en estudio.
\end{abstract}

Palabras Clave:Liderazgo, Rendimiento Académico, coeficiente Rho.

\section{University teacher leadership and academic performance}

\begin{abstract}
The purpose of the research was to determine the relationship between the leadership style of the teachers and the academic performance of the students of the IV cycle of the Faculty of Hotel and Tourism Administration of the National University of Frontera-Peru. The research was analytical, ambispective and of a non-experimental, cross-sectional, correlational design. The data collection was carried out through the use of observation techniques and the survey, with its instruments: technical observation sheets and questionnaire; respectively. The study sample was represented by 14 students. For the statistical analysis, the measures of central tendency were used, such as the arithmetic mean, the variance and the standard deviation. In addition, inferential statistics, Spearman's Rho coefficient, was used to measure the correlation between the teacher leadership variable and the students' academic performance. The results showed that the teachers apply the democratic leadership style, obtaining in the students an average academic performance of 13.26 as acceptable and the coefficient Rho = -0.44 , which indicates the existence of a negative mean correlation between the variables under study.
\end{abstract}

Keywords: Seminar, master class, teaching methods.

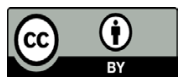




\section{I.INTRODUCCIÓN}

El sistema educativo universitario ha representado cambios significativos en los últimos años. La búsqueda de nuevas propuestas, innovadoras y creativas, son elementos básicos y necesarios para encontrar respuesta a la problemática que tiene relación con la formación profesional y por ende con el rendimiento académico de los estudiantes.

El docente universitario cumple una de las laborales más esenciales de la educación peruana: formar a las futuras generaciones que direccionarán a la sociedad por los caminos del desarrollo y la sostenibilidad. Para este estudio algunos aportes de investigadores [1] han determinado que el nivel de incidencia del estilo de liderazgo desarrollado en tres áreas educacionales: la dirección, los profesores(as), los padres y apoderados evidencian en los resultados académicos de sus alumnos que sólo el área de dirección impacta de manera positiva en los docentes que tienen comportamientos optimistas generando un buen clima de aprendizaje en el aula. Por otro lado [2] se encontró que existe una correlación significativa entre el liderazgo docente y el rendimiento académico, aunado a una alta correlación entre las dos variables, (Rho de Spearman $=0.893$, $\mathrm{p}<$ 0.05) se concluye que el liderazgo docente, entendido como un direccionamiento compartido y pedagógico ejercido por el docente y sumado a la gestión didáctica en el aula, requieren que el docente ejerza un liderazgo democrático y participativo lo que abonaría a la mejora del rendimiento académico de los estudiantes.

La sociedad en su conjunto, espera una formación integral y de calidad en los estudiantes, para lograr profesionales que puedan conducir adecuadamente hacia el camino del desarrollo de la región y el país. El propósito de esta investigación es, contribuir en la tarea de fortalecer las prácticas pedagógicas universitarias a través del conocimiento de la realidad, fomentando el interés en los profesionales que están involucrados en el ámbito de la educación universitaria y de esta manera lograr el desarrollo integral del estudiante. Por lo expuesto, el objetivo de la investigación fue determinar la relación entre el estilo de liderazgo de los docentes y el rendimiento académico de los estudiantes del IV ciclo de la Facultad de Administración Hotelera y de Turismo de la Universidad Nacional de Frontera- Perú.

La presente investigación aplicó el método analítico, diseño no experimental y Transversal; porque se tomó los datos o medidas de las unidades de estudio en una sola oportunidad. La muestra de estudio estuvo representada por 14 estudiantes, cuya selección fue no probabilística intencional; es decir que fueron seleccionados a criterio y conveniencia personal. Así mis- mo se tomó 7 cursos de la currícula del IV ciclo como unidades de estudio. Las técnicas utilizadas fueron la observación directa bibliográfica y la encuesta, con sus instrumentos de fichas de observación y el cuestionario, respectivamente. Para el análisis descriptivo se utilizó la estadística de tendencia central. En cuanto a los resultados obtenidos se evidenció que existe una relación significativa entre el estilo de liderazgo desarrollados por los docentes y el rendimiento académico de los estudiantes del IV ciclo de la Facultad de Administración de Hotelera y de Turismo de la Universidad Nacional de Frontera-Perú.

\section{II.DESARROLLO}

\section{Liderazgo del docente universitario}

El liderazgo es uno de los temas en donde mayor incidencia siguen teniendo los investigadores [3] debido a que se demuestra en la sociedad, organizaciones e instituciones como un factor de éxito [4]; así mismo se ve reflejado en la habilidad de direccionar grupos e individuos para conseguir los objetivos trazados [5]. Se presume en un alto nivel de probabilidad que las organizaciones seleccionan personal poseedores de competencias actitudinales que permitan el avance y evolución de los objetivos institucionales [6] a través de la aplicación de los diferentes estilos de liderazgo.

Se define el liderazgo como la influencia que se ejerce sobre un grupo humano a través del proceso de la comunicación [7] y que se desarrolla en función de las relaciones existentes entre los miembros de una sociedad. Este concepto evoluciona de forma dinámica [8] a medida que se incorporan elementos nuevos que aporten a su desarrollo y que evidencien la incidencia del líder sobre el grupo humano. Es entonces que el estilo de liderazgo [9] es "la manera particular de establecer relaciones interpersonales encaminadas al logro de una o varias metas, desarrollando una actitud de servicio en beneficio de la colectividad a partir de experiencias sociales y educativas adquiridas en el proceso de formación".

Por otro lado, en las organizaciones educativas superiores se concibe como líder al docente que aplica un liderazgo efectivo por medio de la motivación, para administrar eficazmente su rol académico, lo cual hace que los estudiantes se conviertan en seguidores de sus conocimientos de manera que consigan satisfacer sus necesidades académicas [10]. En este sentido se sostiene que el liderazgo está implantado en las instituciones educativas a través del quehacer del docente como líder influyente de la transformación e integración social 
[11].

En entonces que la ausencia de liderazgo [12] evidencia conductas negativas de los estudiantes, entre ellos: comportamientos contradictorios, malas costumbres, falta de respeto, falta de atención, incumplimiento de tareas, rebeldías; es así, como el docente reacciona de forma inadecuada al perder el control del grupo. Y a pesar que el docente se esfuerza por desarrollar un buen liderazgo y mantener la disciplina, necesita actualizarse en el uso de estrategias y herramientas que le permitan desenvolverse de la mejor manera en el aula.

En este sentido se definen tres estilos básicos de liderazgo [13] [14]: el primero consiste en un liderazgo autoritario que comprende autonomía en la toma de decisiones, dirigiendo y controlando el equipo [15]. El segundo denominado liderazgo democrático; aquí se consulta con los subordinados analizando opiniones y a partir de ello, ejercer decisiones [16]. El tercero señalado como Laissez faire; este tipo de liderazgo tiene escasa aportación de ideas y delega en demasía responsabilidades a sus subalternos [4]. Y como cuarto estilo de liderazgo encontramos el carismático [12], que posee la habilidad de atracción del grupo y un elevado desempeño social.

\section{Rendimiento académico}

El rendimiento académico es el resultado cuantitativo o cualitativo [17] de un proceso de evaluación continua [18] desarrollado dentro de la planificación, organización y ejecución de la normativa de enseñanza-aprendizaje en la educación superior [19], de tal forma que involucra factores sociales donde encontramos a la familia [20], los docentes y la misma institución [21]. Cabe resaltar que la búsqueda de información en bases de datos relevantes e incluso redes sociales sirven de apoyo y fortalecen el rendimiento de los estudiantes [22].

Es preciso aclarar que el rendimiento académico es el producto que rinde el estudiante en el campo de la enseñanza [23], alcanzando metas académicas en un lapso de tiempo planteado (semestre académico) y que se ve reflejado en las calificaciones obtenidas. Así mismo, es un indicador de eficacia y calidad [24] en la educación universitaria [25]. Es indispensable señalar que la concentración es un factor de suma relevancia que ayuda al discente a tener un rendimiento óptimo y al mismo tiempo cumplir con los plazos establecidos por el docente [26], de esta forma determinará su continuidad en la carrera profesional y consecuentemente su desarrollo laboral y personal [27].

\section{III.METODOLOGÍA}

La presente investigación aplicó el método analítico debido a la existencia de dos variables: una independiente y la otra dependiente; ambispectivo, (prospectivo y retrospectivo) porque se han tomado los datos de fuentes primarias (encuestas) y de fuentes secundarias (registros de notas promocionales). El diseño fue no experimental, ya que no se manipulan las variables en estudio, solo se limitará a observarlas y medirlas; Transversal, porque se tomará los datos o medidas de las unidades de estudio en una sola oportunidad y el análisis de Correlación de Spearman que es una técnica no paramétrica, que se aplica a aquellos estudios en los que no se cumple con los supuestos de normalidad en los datos y es muy útil en las muestras pequeñas, a través de la comparación de rangos en grupos de sujetos [28]. Este coeficiente es muy útil cuando el número de pares de sujetos (n) que se desea asociar es menor de 30, y permite saber si hay relación entre las variables, cuál es su dirección y que tan fuerte es dicha relación [29].

La muestra de estudio estuvo representada por 14 estudiantes, cuya selección fue no probabilística intencional; es decir que fueron seleccionados a criterio y conveniencia personal. Así mismo se tomó 7 cursos de la currícula del IV ciclo como unidades de estudio. El instrumento utilizado para la recolección fue a través del uso de las técnicas de la observación directa y la encuesta, utilizando como instrumentos las fichas técnicas de observación y el cuestionario. Para la obtención de la información de la variable dependiente, se usaron las Actas de Notas del semestre académico, de la facultad.

Para el análisis descriptivo se utilizó la estadística de tendencia central: la media que nos permitió determinar el promedio de las notas de los estudiantes; la varianza y la desviación estándar que determinó cual es la dispersión de las notas con respecto al promedio y para realizar inferencias se usó la prueba de hipótesis de RHO de Spearman, usando la versión IBM SPSS Statistics 22.0 debido al tamaño de la muestra; menor a 30 (promedio de 14 estudiantes), el mismo que permitió medir la relación entre las variables estudiadas. La correlación mide la asociación [30], no la causalidad, es un método para evaluar la interrelación existente entre un par de variables.

\section{IV.RESULTADOS}

En la Tabla 1 se observa que el estilo de liderazgo de los siete docentes que dan catedra en el IV ciclo de la Facultad de Administración de Hotelería y Turismo es democrático, y carismático y solo dos de ellos aplica el liderazgo de estilo autoritario. 
Tabla 1. Resumen del estilo de líder del docente universitario.

\begin{tabular}{|c|c|c|c|c|c|c|c|}
\hline & \multirow[t]{2}{*}{$\mathrm{N}^{\circ}$ PREGUNTAS } & \multirow[t]{2}{*}{$\begin{array}{l}\text { ESTILOS DE } \\
\text { LÍDER }\end{array}$} & \multicolumn{2}{|c|}{ SI } & \multicolumn{2}{|c|}{ No } & \multirow{2}{*}{$\begin{array}{l}\text { TOTAI } \\
\text { F }\end{array}$} \\
\hline & & & $\mathbf{F}$ & $\% \mathrm{~F}$ & $\mathbf{F}$ & $\% \mathrm{~F}$ & \\
\hline 1. & $\begin{array}{l}\text { El docente permite la participación del estudiante en las } \\
\text { actividades académicas. }\end{array}$ & Democrático & 13 & 100 & 0 & 0 & 13 \\
\hline 1. & $\begin{array}{l}\text { El docente pide la opinión de sus estudiantes para elaboración de } \\
\text { sus exámenes de evaluación, así como el día y hora de su } \\
\text { ejecución. }\end{array}$ & Democrático & 8 & 62 & 5 & 38 & 13 \\
\hline 1. & El docente es accesible y escucha a todos los estudiantes. & Democrático & 11 & 85 & 2 & 15 & 13 \\
\hline 1. & $\begin{array}{l}\text { El docente administra premios y castigos a sus estudiantes según } \\
\text { su comportamiento. }\end{array}$ & Autoritario & 4 & 31 & 9 & 69 & 13 \\
\hline 1. & $\begin{array}{l}\text { El docente, impone sus reglas de conducta en sus actividades } \\
\text { académicas. }\end{array}$ & Autoritario & 2 & 15 & 11 & 85 & 13 \\
\hline 1. & $\begin{array}{l}\text { El docente delega funciones propias de él, al delegado del aula u } \\
\text { otros estudiantes. }\end{array}$ & Laizier & 0 & 0 & 13 & 100 & 13 \\
\hline 1. & $\begin{array}{l}\text { El docente permite las ac ciones no acadé micas (chatear, conversar, } \\
\text { cantar) de los estudiantes en clase. }\end{array}$ & Laizier & 3 & 23 & 10 & 77 & 13 \\
\hline 1. & $\begin{array}{l}\text { El docente muestra interés en las actividades académicas de sus } \\
\text { estudiantes. }\end{array}$ & Carismático & 13 & 100 & 0 & 0 & 13 \\
\hline 1. & $\begin{array}{l}\text { El docente mantiene el orden y respeto con autoridad durante sus } \\
\text { clases. }\end{array}$ & Autoritario & 1 & 8 & 12 & 92 & 13 \\
\hline 1. & $\begin{array}{l}\text { Las decisiones del docente de cómo llevar las clases, es } \\
\text { influenciado por las actitudes de los estudiantes. }\end{array}$ & Laizier & 3 & 23 & 10 & 77 & 13 \\
\hline 1. & E1 docente genera confianza y amistad en sus estudiantes. & Carismático & 13 & 100 & 0 & 0 & 13 \\
\hline 1. & $\begin{array}{l}\text { El docente motiva a sus estudiantes en la participación de las } \\
\text { actividades académicas. }\end{array}$ & Carismático & 12 & 92 & 1 & 8 & 13 \\
\hline & & Prom & 6.92 & 53. & & 46.8 & \\
\hline
\end{tabular}

Fuente: Cuestionario aplicado a los estudiantes de pregrado.

En la tabla 2 para determinar el rendimiento académico de los estudiantes de la Facultad de Administración Hotelera y de Turismo se ha tomado la información de los registros de notas promocionales de los 7 cursos dictados por los docentes. Así mismo, el promedio del rendimiento académico de los estudiantes es de 13. 29 puntos los cuales se encuentran en un nivel "Eficiente Aceptable" [31]. En el DCN [32] las escalas de calificación de 13 se encuentran en un nivel de pro- ceso, es decir está encaminado a lograr un aprendizaje significativo. Para ésta investigación se consideró los siguientes niveles: Deficiente: 0 - 10 puntos, Aceptable: 11 - 13 puntos, Bueno: 14 - 16 puntos, Excelente: 1720 puntos. Así mismo, la varianza tiene el valor de 4.12 y la desviación estándar de 2.03, lo que señala que las notas de los estudiantes se desvían en promedio en un 2.03 puntos de la media calculada. 
Tabla 2. Notas Promocionales de los estudiantes.

\begin{tabular}{ccccc}
\hline NOTAS & $\mathbf{f i}$ & $\mathbf{F i}$ & $\mathbf{h i}$ & $\mathbf{\% h i}$ \\
\hline 7 & 1 & 1 & 0.007 & 0.7 \\
8 & 1 & 2 & 0.007 & 0.7 \\
9 & 4 & 6 & 0.029 & 2.9 \\
10 & 2 & 8 & 0.015 & 1.5 \\
11 & 18 & 26 & 0.131 & 13 \\
12 & 24 & 50 & 0.175 & 17.5 \\
13 & 19 & 69 & 0.139 & 14 \\
14 & 30 & 99 & 0.219 & 22 \\
15 & 17 & 116 & 0.125 & 12.5 \\
16 & 17 & 133 & 0.125 & 12.5 \\
17 & 2 & 135 & 0.014 & 15 \\
18 & 2 & 137 & 0.014 & 15 \\
$1 \sum^{6}$ & $\mathbf{1 3 7}$ & & $\mathbf{1 , 0 0}$ & $\mathbf{1 0 0}$ \\
\hline
\end{tabular}

Fuente: Revisión de actas académicas

En la tabla 3 al realizar la correlación existente entre la variable estilo de liderazgo del docente universitario y el rendimiento académico de los estudiantes del IV ciclo de la Facultad de Administración de Hotelería y
Turismo se puede notar que el valor de correlación es -0.414 , lo que significa que existe una relación significativa entre ambas variables.

Tabla 03. Correlaciones de variables

\begin{tabular}{|c|c|c|c|c|}
\hline \multicolumn{3}{|c|}{ Correlaciones } & \multirow[b]{2}{*}{$\begin{array}{l}\text { ESTILOS DE } \\
\text { LIDERAZGO }\end{array}$} & \multirow[b]{2}{*}{$\begin{array}{l}\text { RENDIMIENTO } \\
\text { ACADÉMICO }\end{array}$} \\
\hline & & & & \\
\hline \multirow{6}{*}{$\begin{array}{l}\text { Rho de } \\
\text { Spearman }\end{array}$} & \multirow{4}{*}{$\begin{array}{l}\text { ESTILOS DE } \\
\text { LIDERAZGO }\end{array}$} & $\begin{array}{l}\text { Coeficiente de } \\
\text { correlación }\end{array}$ & \multirow[t]{2}{*}{1,000} & $-0,414$ \\
\hline & & Sig. (bilateral) & & 0,125 \\
\hline & & & 15 & 15 \\
\hline & & $\begin{array}{l}\text { Coeficiente de } \\
\text { correlación }\end{array}$ & & \multirow{2}{*}{1,000} \\
\hline & \multirow{2}{*}{$\begin{array}{l}\text { RENDIMIENTO } \\
\text { ACADÉMICO }\end{array}$} & Sig. (bilateral) &, 125 & \\
\hline & & $\mathrm{N}$ & 15 & 36 \\
\hline
\end{tabular}

Fuente: Programa informático SPSS aplicado a los datos obtenidos de las variables estudiadas. 


\section{V.CONCLUSIÓN}

La investigación refleja que los estilos de liderazgo más aplicados por los docentes son el democrático y carismático, evidenciando en la práctica pedagógica un dominio integral del grupo en estudio y plena coordinación mutua. Es preciso indicar que los docentes son el prototipo de líder a seguir de los estudiantes durante el pregrado y desarrollo profesional, por tanto deben aplicar el liderazgo en aula y transmitir en los estudiantes ese estilo de dirigir grupos, delegando tareas puntuales a cada uno, fomentando el trabajo colaborativo y en equipo para construir en ellos personalidades consistentes que involucren su participación activa en el desarrollo social de su comunidad, a través de la gestión eficiente, el dialogo y compromiso. Así mismo el liderazgo del docente permite desarrollar con mayor incidencia la práctica académica de los estudiantes, sintiéndose más seguros al participar y actuar frente a los contenidos, y cuyos resultados se evidencian en un rendimiento académico acorde al nivel de pregrado en que se encuentran.

\section{REFERENCIAS}

[1]R. A. P. Vidal, «El liderazgo y su relación con el rendimiento académico. Universidad del Bio Bio,» Chillan, 2008.

[2]M. B. Castro, «El liderazgo docente y el rendimiento académico en el área de persona, familia y relaciones humanas de los alumnos en la I.E. 5117, Jorge Portocarrero Rebaza. Ventanilla, 2014,» Lima, 2015.

[3]F. A. G. Contreras, . E. Navarrete Andrade y W. Suárez Amaya, «Aproximación a los fundamentos teóricos del liderazgo auténtico.,» Revista Venezolana de Gerencia, vol. 22, nº 77, pp. 36-55, 2017.

[4]C. Fernández y N. Quintero, «Liderazgo transformacional y transaccional en emprendedores venezolanos.,» Revista Venezolana de Gerencia, vol. 22, n ${ }^{0}$ 77, pp. 56-74.

[5]L. P. Rejas, C. Araneda Guirriman, A. Bernasconi y . P. Viancos, «Liderazgo, cultura académica y calidad de las universidades: aproximación conceptual y relaciones.,» Revista Venezolana de Gerencia, vol. 23, $\mathrm{n}^{\mathrm{o}} 1$, pp. 184 - 199., 2018.

[6]L. A. Montoya, O. Luna Monterrosa, S. Navarro Lobo y B. Salas Muñoz, «Estilos de liderazgo de la alta dirección en industrias exportadoras de Barranquilla,» Revista Venezolana de Gerencia, vol. 24, nº 86, pp. 575591., 2019.

[7]D. M. E. Barbosa y A. Hurtado Ayala, «Influencia de los estilos de liderazgo en el desempeño de las empresas exportadoras colombianas,» Estudios Gerenciales, vol. 32, p. 137-145, 2016.
[8]J. Zuzama, «Estilos de liderazgo según Kurt Lewin y análisis de un caso real,» 2017.

[9]O. Gonzáles y L. Gonzáles, «Impacto del estilo de liderazgo del docente universitario en el rendimiento académico del estudiante,» Revista Multiciencias, vol. 14, no 04, pp. 401-409, 2014.

[10]Y. Alvarado, A. T. Prieto Sánchez y D. Betancourt, «Liderazgo y motivación en el ambiente educativo universitario,» Revista Electrónica "Actualidades Investigativas en Educación”, vol. 9, nº 3, pp. 1-18, 2009.

[11]G. P. Ortega, L. Guarín Herrera y G. Romo Morales, «Liderazgo transformacional en los docentes universitarios desde la perspectiva estudiantil. Estudio de caso: universidad nacional de Colombia, sede Medellín,» SABER, CIENCIA Y Libertad, vol. 10 , nº 1, pp. 203-2018, 2015.

[12]G. Y. A. Tirado, «Liderazgo docente y disciplina en el aula,» Quetzaltenango, Guatemala, 2014.

[13]L. K., «Experiments in social space,» pp. 71-83, 1939.

[14]J. M. V. Vergés, Función directiva, España: Universitat Autònoma de Barcelona, 1999.

[15]J. B. S. Reyes y . L. Barraza Barraza, «Percepciones sobre liderazgo,» Revista Ra Ximhai, vol. 11, $\mathrm{n}^{\circ}$ 4, pp. 161-170, 2015.

[16]M. Villalva y Isidro Fierro, «El liderazgo democrático: Una aproximación conceptual,» INNOVA Research Journal, vol. 2, nº 4, pp. 155-162, 2017.

[17]P. L. Vera, L. R. Trelles Pozo y M. M. Mogollón Taboada, «Asertividad y rendimiento académico en estudiantes de la facultad de ciencias administrativas de la Universidad Nacional de Piura,» UCV-SCIENTIA, vol. 11, $n^{\circ} 1$, pp. 13-20, 2019.

[18]K. Perez, «Influencia de la función ejecutiva en el rendimiento académico de estudiantes universitarios. Caso Fundación Universitaria Tecnológico Comfenalco,» Revista Espacios, vol. 40, no 8, p. 7, 2019.

[19]R. A. A. Sauceda y Á. E. Rafael Sánchez, , «Estrategias de aprendizaje y rendimiento académico universitario: Una mirada desde los estudiantes de tecnológicos públicos,» Revista Venezolana de Gerencia, vol. 24, $n^{\circ}$ 87, pp. 938-953., 2019.

[20]M. E. G. Salinas, «Factores Socioeconómicos y pedagógicos que inciden en el Rendimiento académico en estudiantes de la carrera de Ciencias Sociales, UNAM - CUR Matagalpa, durante el I semestre, 2008,» 2010.

[21]M. J. C. López, A. Llanes Castillo, A. A. Peña Maldonado y . J. Cruz Casados, «Estrategias para potenciar el aprendizaje y el rendimiento académico en estudiantes universitarios,» Revista Venezolana de Gerencia, vol. 25, nº 90, pp. 579-594, 2020.

[22]R. Martelo, I. Jiménez Pitre y P. M. Martelo, «). In- 
cidencia de las redes sociales en el rendimiento académico de los estudiantes de la universidad de La Guajira (Colombia),» Revista Espacios, vol. 38 , nº 45, p. 24, 2017.

[23]E. G. D. Caballero y . A. CONTRERAS Orozco, «Estilos de vida y rendimiento academico de adolescentes escolarizados del departamento de Sucre. Colombia,» Espacios, vol. 41, nº 11, p. 28, 2020.

[24]M. Custodio, C. Espinoza , C. Baltazar, . R. Montalvo S. Ochoa y R. Peñaloza, «Rendimiento académico de estudiantes de medicina humana según modalidad de admisión en la Universidad Nacional del Centro del Perú,» Espacios, vol. 41, nº 9, p. 24, 2020.

[25]M. Rezazadeh y M. Tavakoli, «Investigating the Relationship among Test Anxiety, Gender, Academic Achievement and Years of Study: A Case of Iranian EFL University Students,» English Language Teaching, vol. 2, no 4, pp. 68-74, 2014.

[26]E. Coneo, C. Martínez y E. Amed, «Atención visual y auditiva y su relación con el rendimiento académico en estudiantes de secundaria,» Espacios, vol. 40, n ${ }^{\circ} 19$, p. 29, 2019.

[27]M. Redondo y L. Jiménez, «Autoconcepto y rendimiento académico en estudiantes de secundaria en la ciudad de Valledupar-Colombia,» Espacios, vol. 41, $\mathrm{n}^{\mathrm{o}}$ 9, p. 17, 2020.

[28]P. Torres, «Acerca de los enfoques cuantitativo y cualitativo en la investigación educativa cubana actual,» Revista científico pedagógica, vol. 2, no 34, pp. $1-5,2016$.

[29]R. Hernández, Metodología de la Investigación, México: Mc Graw-Hill, 2006.

[30]S. Ross, Introducción a la Estadística. Reimpresión, Barcelona. España: Editorial Reverté S.A., 2008.

[31]J. Acuña, "Autoestima y Rendimiento Académico de los estudiantes del X Ciclo 2012 - II de la Escuela Académica Profesional de Educación Primaria y problemas de aprendizaje de la Universidad Nacional José Faustino Sánchez Carrión - Huacho.,» Huacho, 2013. [32]J. Domínguez y . C. Tamayo, «Intervenciones educativas con estrategias didácticas bajo el enfoque Socio cognitivo orientadas al desarrollo del aprendizaje en los estudiantes de Educación Básica regular de Perú.,» Chimbote, 2011.

[33]F. Chávez, «El liderazgo personal e interpersonales docentes y estudiantes de enfermería de la U.N.M.S.M,» Lima, 2007.

[34]A. Mondragón, «Uso de la correlación de Spearman en un estudio de intervención en fisioterapia.,» Rev. Mov. Cient., vol. 8, nº 1, pp. 98-104, 2015.

[35]L. Suarez, «Desempeño docente y rendimiento académico en el área de matemáticas en la I.E. Carlos J.
Arosemena Tola del cantón de la provincia del Guayas Ecuador 2018"..,» 2018.

[36]J. L. L. Castilla, «La Enseñanza Universitaria, los Recursos Didácticos y el Rendimiento Académico de los estudiantes de la E.A.P de Educación de la Universidad Nacional Mayor de San Marcos,» 2012.

[37]V. Martínez, « Factores condicionantes del rendimiento escolar y perfil de alumnos con alto rendimiento.,» Madrid. España, 1997.

[38]C. Muñoz y J. Guzmán, «Calidad docente e insumos físicos de las escuelas como factores del rendimiento escolar en educación primaria.,» México, 1991. [39]R. Hernández

\section{RESUMEN CURRICULAR}

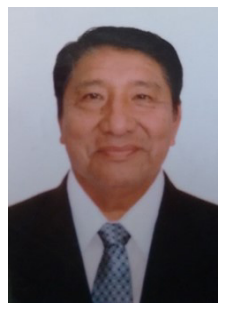

Mario Villegas Yarleque, Economista con maestría en Ciencias de la Educación Superior, con experiencia en el sector privado en empresas de transporte de pasajeros y sector público, en gobiernos locales y regionales; con 14 años de experiencia académica en universidades públicas y privadas. Actualmente me desempeño como docente auxiliar a tiempo completo en la Universidad Nacional de Frontera en la Facultad de Ingeniería Económica desde Marzo del 2017 hasta la actualidad, en calidad de docente nombrado. E-mail: mvillegas@unf.edu.pe

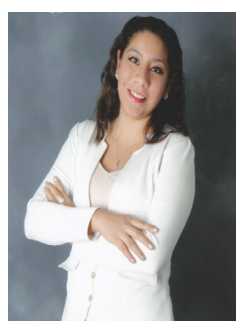

Gretel Fiorella Villegas Aguilar, Docente mentor a cargo de desarrollar nuevos modelos de negocios sostenibles con los estudiantes y ayudarlos en su formación académica profesional en Administración de Empresas en la Universidad Privada del Norte. Grado de Magister con mención en Docencia Universitaria e Investigación Pedagógica. Licenciada en Administración de empresas. Estudios de especialización en creación de nuevos modelos de negocios. E-mail: gretel.villegas@upn.pe

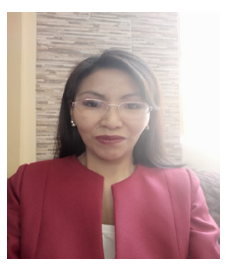

Apaza Panca Cynthia Milagros,Licenciada en Administración y Marketing, Contador Público, Magíster en Economía con mención en Planificación y Gestión Pública, y Estudios Concluidos de Doctorado en Economía y Políticas Públicas. Docente Auxiliar de la Facultad de Administración Hotelera y Turismo de la Universidad Nacional de Frontera de Sullana, Piura. Experiencia Docente en universidades públicas y privadas, con interés en las áreas de: marketing, emprendimiento, gestión pública y políticas públicas en turismo. Investigadora CONCYTEC, Grupo: María Rostworowski, Nivel II. Email: capaza@unf.edu.pe 
Susana Soledad Chinchay Villarreyes, Doctora en Gestión e Investigación de la Educación. Cerca de 28 años de experiencia laboral en la docencia, de los cuales 11 años han sido dedicados a la docencia universitaria. Profesional proactiva y comprometida con el trabajo, capacidad de liderazgo y de gestión.

E-mailschinchay@unf.edu.pe

Priscila E. Luján-Vera, Doctorada en Ciencias de la Educación. Investigadora CONCYTEC, Grupo: María Rostworowski, Nivel II. Experiencia profesional enfocada en la planificación y ejecución de proyectos de Innovación en Gestión Social, Turística y Empresarial. E-mail: priscilavera930@gmail.com 